Trauma Berufskrankh 2005 · 7[Suppl 1]:S153-S155 DOI 10.1007/s10039-004-0913-6

Online publiziert: 14. Juli 2004

(c) Springer-Verlag 2004

M. Müller · L. Besch · H.-J. Egbers

Klinik für Unfallchirurgie, Universitätsklinikum Schleswig-Holstein, Campus Kiel

\title{
Verletzungen des Sprungbeins
}

Im Stand und beim Gang erfolgt die axiale Lastübertragung von der Tibia in den Talus. Von dort wird sie über das Talonavikulargelenk in die mediale Fußsäule, über die unteren Sprunggelenke zum Fersenbein und über das Kalkaneokuboidalgelenk in die laterale Fußsäule eingeleitet. Talokruralgelenk und die unteren Sprunggelenke sind einheitlich an einer kardanähnlichen Bewegung beteiligt, durch die die Körperlast bei den Stand- und Gangvariationen von der frontalen auf die horizontale Ebene übertragen wird und umgekehrt [7].

\section{Unfallmechanismus}

Talusverletzungen entstehen überwiegend infolge von Hochrasanztraumen, bedingt durch Verkehrsunfälle und Stürze aus großer Höhe. In der Regel sind komplexe multidirektionale Krafteinwirkungen auf den Fuß notwendig, Rotations- und Stauchungsmechanismen sind führend.

Der im Augenblick der deformierenden Energie dorsal extendierte Fuß stellt biomechanisch die Grundlage für die häufige Talushalsfraktur dar, die etwa 50\% aller Sprungbeinbrüche ausmacht. Dabei verkeilt sich die distale Tibiavorderkante im Talushals und schert diesen ab. Das Sustentaculum talare fungiert dabei als Hypomochlion.

Die Korpusfraktur entsteht bei plantar flektiertem Fuß. Der Sprungbeinkörper wird nach dorsal durch die hintere Tibiakante abgedrängt. Bei hoher Gewalteinleitung können zusätzlich die Bandverbindungen zwischen Sprung- und Fersenbein zerreißen, sodass der frakturierte Sprungbeinkörper unter der Tibia rotiert und sich dorsal hinter dem Fersenbein verhakt.
Die seltenen isolierten Taluskopffrakturen entstehen am häufigsten bei $\mathrm{Ab}$ - und Adduktionstraumen mit kombinierter rotatorischer Komponente des Rückfußes gegenüber der Fußwurzel unter axialer Krafteinwirkung.

\section{Klassifikationen}

Die Einteilung aller Talusfrakturen erfolgt nach Marti [4], die der Talushalsfrakturen nach Hawkins [3], erweitert durch Canale u. Kelly [2]. Zwipp [11] klassifizierte die Verletzung nach der Anzahl der durch die Fraktur des Talus und Dislokationen beteiligten Gelenke. Aus detaillierten Angaben zur Verletzungsschwere und zum Grad der Fragmentverschiebung soll das Nekroserisiko abzuschätzen sein. Periphere Verletzungen des Talus sind bezüglich der Nekroserate von $5 \%$ prognostisch günstig, wohingegen zentrale Mehrfragmentfrakturen mit bis zu $50 \%$ das höchste Nekroserisiko aufweisen.

Bei der Evaluation des Schweregrads ist davon auszugehen, dass in etwa $50 \%$ eine isolierte Talusfraktur vorliegt und ebenso häufig mit einer Komplexverletzung der Fußwurzel zu rechnen ist [8]. Das wird um so deutlicher angesichts der Tatsache, dass die Talusbruchlast mit bis zu $4000 \mathrm{~N}$ einer hohen Traumaenergie bedarf.

\section{Bildgebende Diagnostik}

Die nativröntgenologische Untersuchung der Fußwurzel ist durch vielfache Überlagerungen knöcherner Konturen, Skelettvariationen sowie eine große individuelle Schwankungsbreite der anatomischen Formen erschwert. Für den Traumatologen ist dies für die Differenzialdiagnose auf die große Variabilität dieses Gefäßsystems zurückgeführt werden [6]. 
„Fraktur oder Anomalie“ von praktischer Bedeutung.

Röntgen. Führen Anamnese, Unfallmechanismus sowie klinisches Erscheinungsbild mit Hämatom, Schwellung, Verrenkungsstellung und lokalem Druckschmerz zur Verdachtsdiagnose einer Talusläsion, gehören seitliche und dorsoplantare Röntgenaufnahmen sowie die Brodén-Projektion für das untere Sprunggelenk zum Standard, um die Diagnose zu sichern.

Computertomographie. Eine enge Kooperation zwischen Radiologen und Traumatologen ist erforderlich, um die Frage des operativen Zugangs (anteromedial, posterolateral, bilateral, median) und der Frakturpathologie zu klären und die mehrdimensionalen Nachverarbeitungen aus präoperativ angefertigten, axialen CT-Datensätzen zu analysieren. Auch postoperativ ist eine CT anzustreben, um Subluxationen und Gelenkinkongruenzen nach Reposition feststellen und in einem sekundären Eingriff beheben zu können.

Magnetresonanztomographie. Sie wird bei persistierenden Beschwerden notwendig. Eine posttraumatische und postoperative partielle oder totale Talusnekrose können so frühzeitig erkannt werden, falsch-negative Untersuchungsergebnisse sind möglich. Um MRT-Artefakte zu minimieren, werden für Sprungbeinoperationen inzwischen ausnahmslos Titanimplantate verwendet.

\section{Indikationen und Therapieverfahren}

Ziel der Frakturbehandlung ist die exakte anatomische Wiederherstellung der knöchernen Strukturen zur Vermeidung von Inkongruenzen. Eine möglichst frühzeitige Rekonstruktion soll die Gefäße entlasten und eine schnelle Revaskularisation ermöglichen. Frühfunktionelle Behandlung des gesamten Fußes sowie des oberen und der unteren Sprunggelenke wird angestrebt.

Periphere Frakturen mit unverschobenen kleinen Fragmenten können konservativ behandelt werden. Größere periphere Fragmente sollten mit Kleinfragmentschrauben oder resorbierbaren Stiften refixiert werden [9]. Die konservative Therapie kann bei allen nicht dislozierten Talus- hals- und Körperfrakturen durchgeführt werden. Das Ausmaß einer evtl. vorliegenden und nativradiologisch nicht erkennbaren Dislokation ist dabei entsprechend dem oben angegebenen Diagnostikalgorithmus sorgfältig zu prüfen. Müller beschrieb bei 44 Talusfrakturen, die in $64 \%$ konservativ behandelt wurden, in immerhin $43 \%$ die Komplikation der Talusnekrose [5].

Die frühzeitige funktionelle Behandlung von Gelenkfrakturen stellt heute ein zentrales Behandlungskonzept dar, sodass auch undislozierte Talusfrakturen osteosynthetisch versorgt werden sollten.

Dislokation und zentrale Frakturlokalisation steigern die Nekrosegefahr. Aus diesem Grund sollten alle dislozierten Frakturen operativ versorgt werden [10]. Die operative Stabilisierung zum frühest möglichen Zeitpunkt wird angestrebt. Im eigenen Patientengut bestand ein hoher Anteil an verzögert zu versorgenden Verletzungen durch Zuverlegungen primär auswärtig versorgter Patienten. Bei dieser $\mathrm{Pa}$ tientengruppe kam es nahezu zu einer Verdopplung der Nekrosehäufigkeit [1].

Zusatzverletzungen am ipsilateralen Fuß kompromittieren den Weichteilmantel, sodass in diesen Fällen nicht selten eine sekundäre Osteosynthese erforderlich wird. Als Primärmaßnahme ist hier die geschlossene Reposition mit Anlage eines transfixierenden Fixateur externe bis zur Erholung des Weichteilmantels anzusehen.

Bei der Typ-II-Fraktur nach Hawkins besteht eine Dislokation des Halsfragments. Die Stabilisierung erfolgt über einen anteromedialen Zugang durch 2 parallel eingebrachte kanülierte Titanspongiosazugschrauben der Stärke 3,5 mm. Bei den TypIII-Frakturen mit Dislokation des Taluskörpers soll der Perfusionsschaden durch eine sofortige Operation, $d . h$. innerhalb von $6 \mathrm{~h}$ nach Trauma, zu verbessern sein. Eine Innenknöchelosteotomie kann zur $\mathrm{Zu}$ gangserweiterung erforderlich werden. Bei Typ-III- und -IV-Verletzungen ist additiv mitunter eine Transfixation zu empfehlen.

Der dorsolaterale paraachilläre Zugang wird vorrangig für Versorgungen des Processus posterior tali und distaler Korpusfrakturen genutzt. Der bilaterale Zugang mit anschließender Osteosynthese ist den Mehrfragment- und Luxationsfrakturen mit Abbruch des Processus fibularis tali vorbehalten.
Operationsverfahren wie die temporäre Spickdrahtarthrodese und die tibiometatarsale Transfixation können zur zusätzlichen Stabilisierung mitunter hilfreich sein. Trotzdem kann der traumatogene Gefäßschaden häufig nur zum Teil kompensiert werden, und es zeigt sich deutlich, dass auch durch eine ideale anatomische Reposition Komplikationen wie Nekrose und Arthrose nicht sicher zu verhindern sind [1].

Bei vollständigen Talusluxationen und Trümmerfrakturen des Sprungbeins kann in Ausnahmefällen die primäre Arthrodese indiziert sein, um dem Verletzten lange Behandlungszeiten mit den bekannten Komplikationen zu ersparen.

Bei Kontraindikation für eine operative Versorgung erfolgt die Immobilisation der verletzten Extremität für 6 Wochen.

\section{Nachbehandlung}

Sie wird kontrovers diskutiert. Es werden Entlastungszeiten der betroffenen Extremität von bis zu 12 Monaten angegeben.

Wir streben eine möglichst frühe funktionelle Therapie an. Postoperativ werden die Patienten nach konsequenter Hochlagerung und Kühlung ab dem 2. postoperativen Tag passiv mittels Motorschiene beübt. Nach Konditionierung der Weichteile erfolgt die Mobilisierung an Unterarmgehstützen mit einer Teilbelastung mit Fußsohlenkontakt und Abrollbewegung für insgesamt 8-12 Wochen. Vollbelastung wird bei knöcherner Konsolidierung nach 4 Monaten angestrebt.

Die Durchblutungssituation des Talus und die typische Komplikation der vaskulären Nekrose insbesondere bei den Frakturtypen III und IV nach Marti werden durch MRT kontrolliert. Als seltene Komplikationen sind sekundäre Dislokationen und Taluspseudarthrosen zu nennen.

\section{Diskussion}

Talusluxationen und Luxationsfrakuren sind seltene Verletzungen. Sie sind als Notfall zu behandeln. Nach frühzeitiger exakter Diagnosestellung durch Röntgenspezialaufnahmen und einem fakultativ durchgeführten präoperativen CT erfolgt in Kenntnis der Klassifikation eine frühzeitige anatomische Reposition. Sie sollte offen durchgeführt werden, um eine 
definitive übungsstabile Osteosynthese durch Verschraubung mit kanülierten 3,5mm-Titanschrauben vornehmen zu können. Die Anzahl der Repositionsversuche muss gering gehalten werden. Die Nekroserate kann durch frühzeitige operative Intervention gesenkt werden. Es können keine Aussagen über das Eintreten einer Talusnekrose getroffen werden. Die Vitalität des Sprungbeins kann im postoperativen Verlauf durch MRT geprüft werden.

Eine frühe funktionelle Behandlung mit Teilbelastung zur Optimierung der Gelenktrophik und Knochenstrukturierung zeigt keine Nachteile gegenüber der Langzeitentlastung.

\section{Korrespondierender Autor Dr. M. Müller}

Klinik für Unfallchirurgie, Universitätsklinikum Schleswig-Holstein, Campus Kiel, Arnold-Heller-Straße 7, 24105 Kiel E-Mail:muellerm@unfchir.uni-kiel.de

Interessenkonflikt: Der korrespondierende Autor versichert, dass keine Verbindungen mit einer Firma, deren Produkt in dem Artikel genannt ist, oder einer Firma, die ein Konkurrenzprodukt vertreibt, bestehen.

\section{Literatur}

1. Besch L, Drost J, Egbers HJ (2002) Die Behandlung der seltenen Talusluxationsfrakturen. Unfallchirurg 105: 595-601

2. Canale S, Kelly F Jr (1978) Fractures of the neck of the talus. Long-term evaluation of seventy-one cases. J Bone Joint Surg Am 60: 143

3. Hawkins $L$ (1970) Fractures of the neck of the talus. J Bone Joint Surg Am 52: 991-1002

4. Marti R (1978) Talus- und Calcaneusfrakturen. In: Weber B (Hrsg) Die Frakturenbehandlung von Kindern und Jugendlichen. Springer, Berlin Heidelberg New York

5. Müller K (1978) Talusfrakturen - Ergebnisse Bochum. In: Burri C (Hrsg) Verletzungen des oberen Sprunggelenkes. Hefte Unfallheilkd 131: 218

6. Mullfinger G, Trueta J (1970) The blood supply of the talus. J Bone Joint Surg Br 52: 160-167

7. Pisani G (1998) Fußchirurgie. Thieme, Stuttgart New York, S 41-46

8. Schuind F, Andrianne Y, Burny F et al. (1985) Komplikationen nach Talustrauma. Aktuelle Traumatol 15: 82-88

9. Schulze W, Richter J, Klapperich T et al. (1998) Funk tionsergebnisse nach operativer Therapie von Talusfrakturen. Chirurg 69: 1207-1212

10. Zwipp H (1993) Severe foot trauma in combination with talar injuries. In: Tscherne $\mathrm{H}$, Schatzker J (eds) Major fractures of the pilon, the talus and the calcaneus. Springer, Berlin Heidelberg New York, pp 123-135

11. Zwipp H (1994) Die Chirurgie des Fußes. Springer, Berlin Heidelberg New York, S 86-99
Trauma Berufskrankh $2005 \cdot 7[$ Suppl 1]:S153-S155

DOI 10.1007/s10039-004-0913-6

(c) Springer-Verlag 2004

\section{Müller $\cdot$ L. Besch $\cdot$ H.-J. Egbers}

\section{Verletzungen des Sprungbeins}

\section{Zusammenfassung}

Sprungbeinverletzungen sind seltene, aber schwere Verletzungen. Aufgrund der Anatomie handelt es sich meist um Gelenkfrakturen. Talushalsfrakturen machen etwa $50 \%$ aller Sprungbeinbrüche aus, es folgen die Korpusfraktur und die seltene Taluskopffraktur. Die sich aus Anamnese, Unfallmechanismus und klinischem Erscheinungsbild ergebende Verdachtsdiagnose Talusläsion wird durch Röntgenaufnahmen gesichert. Die CT dient zur Klärung des operativen Zugangs, der Frakturpathologie, der mehrdimensionalen Nachverarbeitung sowie der postoperativen Kontrolle. Die MRT wird bei persistierenden Beschwerden not-

\section{Injuries to the ankle bone}

\section{Abstract}

Injuries to the talus are rare, but serious. The anatomy of this region means that most injuries affecting it are joint fractures. Fractures of the neck of the talus account for about $50 \%$ of all talar fractures, followed by fractures of the corpus and then fractures of the talar head, which are rare. A tentative diagnosis of a talar lesion can be suggested by the history, the mechanism of injury and the clinical picture and then confirmed by an X-ray examination. CT can indicate the best operative technique, the fracture pathology, and the best multidisciplinary aftercare and is also helpful in postoperative monitoring. MRT is necessary when symp- wendig. Die Gelenkfrakturen sollten möglichst frühzeitig behandelt werden, um die häufige Komplikation Nekrose gering zu halten. Es können jedoch keine Aussagen zur Wahrscheinlichkeit des Auftretens derselben gemacht werden. Die vielfältigen Therapiemöglichkeiten werden dargestellt. Hinsichtlich der Nachbehandlung streben wir eine möglichst frühe funktionelle Therapie an.

\section{Schlüsselwörter}

Talushalsfraktur · Korpusfraktur . Taluskopffraktur · Frühzeitige funktionelle Therapie

toms persist. Joint fractures must be treated as soon as possible to restrict the likelihood and the severity of necrosis; though this is a frequent complication it is impossible to estimate how likely it is in a particular case. The many treatment options available are presented. In the aftercare we are concerned to implement functional therapy as early as possible.

\section{Keywords}

Fracture of the talar neck - Fracture of the body of the talus . Fracture of the talar head. Early functional therapy 